\title{
Dizziness and benign paroxysmal positional vertigo among retirement home residents: a cross-sectional descriptive and interventional study
}

Rainer Müller ${ }^{1,2}$, Peter Zangger ${ }^{1}$, Dominik Straumann ${ }^{1,2,3}$ and Stefan Y. Bögli1 ${ }^{1,2,3^{*}}$

\begin{abstract}
Background: The prevalence of dizziness increases with age. We aimed to determine the point prevalence of dizziness and, in particular, of benign paroxysmal positional vertigo (BPPV) among retirement home residents. Furthermore, we aimed to evaluate the efficacy of a 2-axis turntable based BPPV treatment.

Methods: We contacted all large retirement homes in or around the city of Zurich (Switzerland). 10 retirement homes (with a total of 536 residents) agreed to participate in this study. 83 rejected inquiries by residents led to a potential study population of 453 residents. After a structured interview evaluating the presence and characteristics of dizziness, all willing patients were tested for positional vertigo and nystagmus on a portable and manually operated 2-axis turntable that was transported to the retirement home. Testing consisted of the Dix-Hallpike and supine roll maneuvers to both sides. Participants were immediately treated with the appropriate liberation maneuver whenever BPPV was diagnosed. Otherwise, taking the resident's medical history, a neuro-otological bedside examination, and a review of the available medical documentation was used to identify other causes of dizziness.
\end{abstract}

Results: Out of the 453 residents, 75 (16.6\%; average age: 87.0 years; $68 \%$ female) were suffering from dizziness presently or in the recent past and gave their consent to participate in this study. Among the participants tested on the turntable $(n=71)$, BPPV was present in $11.3 \%$ (point prevalence). Time-related properties, triggering factors and qualitative attributes of vertigo or dizziness were not significantly different between the dizzy participants with and those without BPPV. In all BPPV patients, appropriate liberation maneuvers were successful.

Conclusions: BPPV could be demonstrated in about one tenth of retirement home residents with dizziness or recent dizziness. Such point prevalence of BPPV translates to a much higher yearly prevalence if one assumes that BPPV is not present on every day. Our finding suggests that retirement home residents suffering from dizziness should be regularly tested for BPPV and treated with appropriate liberation maneuvers, ideally on turntable to reduce strain.

Trial registration: ClinicalTrials.gov Identifier NCT03643354.

Keywords: vertigo, Dizziness, Benign paroxysmal positional vertigo, Epidemiology, Retirement home

${ }^{*}$ Correspondence: stefanyu.boegli@usz.ch

${ }^{1}$ Department of Neurology, University Hospital Zurich, Zurich, Switzerland

Full list of author information is available at the end of the article

\section{Background}

Dizziness is one of the most common medical problems, especially in the older population. Various studies [1-3] indicate a steady increase of dizziness with age. The prevalence of dizziness in people who exceeded the age of 65 
is around 30\% [2, 3] and rises to almost 50\% [3] in those older than 85 . In particular, among nursing home residents prevalence of dizziness has been found to be up to almost $45 \%$ (peaking between 80 and 90 years of age) [4].

Etiologies of dizziness include peripheral and central vestibular, cardio-vascular, ocular motor, visual, and somatosensory disorders. In most cases, a well-focused patient history, a small set of neuro-otological bedside tests and a orthostatic evaluation suffice for identifying the primary causes of dizziness [5]. Neuro-otological bedside tests [6] should always include diagnostic maneuvers for benign paroxysmal positional vertigo (BPPV) [7].

Among older people the most frequent origin of dizziness or vertigo is BPPV [8], accounting for about one third of diagnoses $[1,9]$. Typically, the first episode of BPPV occurs between 49 and 60 years [8, 10-12]. Despite the severe impact of BPPV on the quality of older individual's lives $[1,13,14]$ and the associated higher risk of falls [3, 15-17], BPPV often remains unrecognized $[9,18]$ and therefore the cause of dizziness remains unclear [9, 19]. Successful therapeutic maneuvers in older patients with BPPV lead to a significant reduction of falls $[16,17]$, thus the underdiagnosing of BPPV needs to be urgently addressed.

Pathophysiologically, there are a variety of reasons why the prevalence of BPPV increases with age. Both osteoporosis as well as vitamin $D$ deficiency have been found to be more prevalent in patients with BPPV $[20,21]$. Both lead to a disruption of the calcium metabolism and have thus been implicated in easing fragmentation or displacement of otoconia [22]. Prolonged recumbancy (e.g. due to impaired mobility) might also facilitate detachment of otoconia [23]. Lastly, older patients have higher recurrence rates of BPPV even after successful repositioning [24].

In otherwise healthy individuals, BPPV can easily be diagnosed with the provocation maneuvers, e.g., DixHallpike and supine roll maneuvers [7]. However, in frail patients - who are mostly older individuals - the correct performance of the maneuvers at the bedside might be hampered, amongst other reasons, by stiffness, pain, and angst [25]. These factors may also lead to hesitations by the treating physicians. Hence, BPPV in older dizzy patients is probably more prevalent than previously thought. The highly effective therapeutic maneuvers, e.g., Epley, Sémont and Gufoni liberation maneuvers, put even more strain on frail patients when performed at the bedside. Thus, to omit neck and head movements relative to the trunk, head rotations relative to gravity, as required by both the provocation and liberation maneuvers, they are best accomplished in frail patients by whole-body rotations on a turntable [26]. Furthermore, liberation maneuvers performed on mechanical turntables might be more successful than classical manually performed maneuvers [27, 28]. The effectiveness of liberation maneuvers using mechanical turntables in retirement home residents has, to our best of knowledge, not been reported yet.

Using a manually driven 2-axis turntable we aimed to determine the point prevalence of BPPV in older patients suffering from dizziness at the day of testing or in the recent past. The turntable is portable, which allowed us to do the testing at the patients' retirement homes. We also monitored the effectiveness of liberation maneuvers that were performed whenever BPPV was diagnosed. Secondarily, we aimed to evaluate whether there were significant differences in the presentation of symptoms between patients with BPPV and patients with dizziness of other origins.

\section{Methods}

The study protocol was approved by the local ethics committee (cantonal ethics commission Zurich, BASEC-Nr. 2018-02017) and was in accordance with the ethical standards laid down in the 2013 Declaration of Helsinki for research involving human subjects. Informed consent was obtained from each subject - for both study participation and for publication of identifying images in an online open-access publication. The clinical trial data and the results are reported on clinicaltrials.gov (Identifier: NCT03643354).

\section{Recruiting process}

This study on older individuals living in retirement homes was performed at 10 institutions in the Canton of Zürich, Switzerland, between 2018 and 2020. During the recruiting process (Fig. 1), we contacted the management of 73 retirement homes and asked whether we could visit their facilities with our "Schwindelbus" containing the the "Rotundum" turntable (Fig. 2). This van would transport the portable 2-axis turntable to the respective retirement home and set up in a larger area within the facility, typically a recreation room. There, any resident with present or recent dizziness would be offered diagnostic and possibly therapeutic BPPV maneuvers on the turntable. This service would be free of charge. The anonymized data would be analyzed scientifically with ethical permission. Surprisingly, the managements of 63 retirement homes were not interested or did not respond, despite repeated enquiry. Reasons for disinterest included the rejection to participate in research, the notion that dizziness was not a major problem among the residents, and the fact that the caregivers were too busy.

The managements of 10 retirement homes, however, agreed to participate. All retirement home residents (536 residents) were informed of the upcoming visit by 


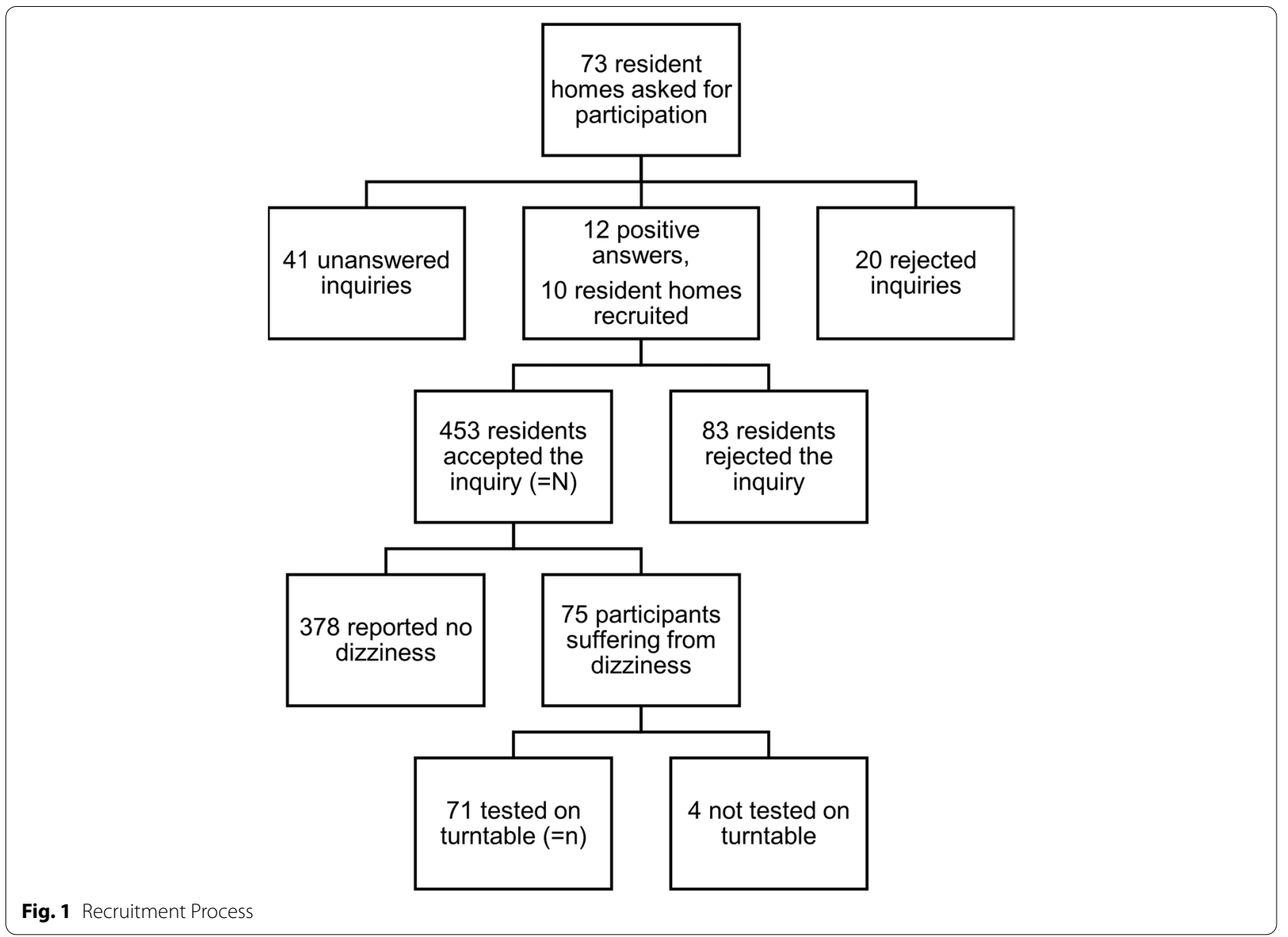

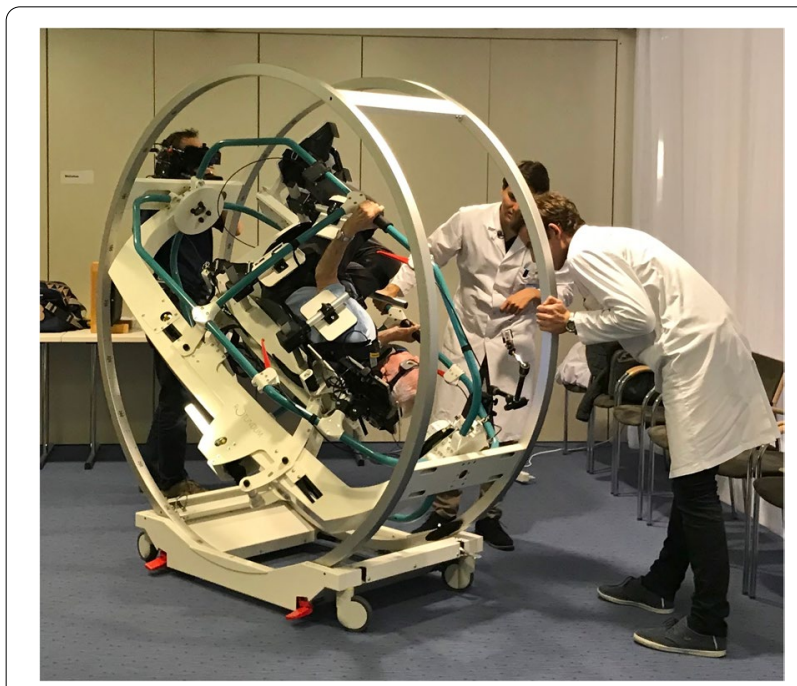

Fig. 2 The "Rotundum" turntable in action the "Schwindelbus" and about the opportunity to receive specialist advice on the topic of dizziness.

All residents who agreed to inclusion were evaluated for the presence of dizziness. Residents who experienced dizziness presently or in the recent past (defined as having occurred at least once within the last 3 months) were interviewed by one of the co-authors (R.M.) based on a questionnaire covering major aspects of their dizziness and its impact on their daily life. Furthermore, age and gender were noted. The questionnaire (translated from German to English) used can be found as a supplement of this paper. Dizziness was enquired using the German word "Schwindel", which encompasses a variety of symptoms including subjective imbalance, illusion of motion (of either the body or the surrounding), as well as lightheadedness, commonly appearing together with different degrees of nausea. Different aspects of dizziness including persistence (i.e. chronic persistent versus recurrent attacks), duration (i.e. up to few minutes versus hours to days), number (i.e. multiple times daily versus once daily down to less than once per month), interictal symptoms (i.e. minor dizziness versus no dizziness), presence of 
triggering factors (i.e. lying down, standing up quickly, turning in bed, reclination of the head), characteristic of dizziness (turning versus swaying), as well as dizzinessinduced nausea or vomiting were inquired. Impairment of quality of life was queried both on a completely subjective level (i.e. does the dizziness impair your quality of life) as well as depending on different daily activities (including activities performed while lying, sitting or standing). Residents who were unable to answer the structured interview questions due to cognitive impairment were excluded from the study. Altogether, 75 out of 453 residents addressed suffered from dizziness or vertigo (Fig. 1).

\section{"Schwindelbus" visit}

Within 1 week after the structured interview, participants were examined on a manually driven 2-axis turntable (Rotundum R1, prolim $\mathrm{GmbH}$ ). Of the 75 individuals 4 could not be tested on the turntable, as they were not able to be present on the day of examination or were afraid of the procedure. 71 residents $(=n)$ underwent diagnostic BPPV provocation maneuvers on the turntable. These were the Dix-Hallpike maneuver [29] (whole-body rotation 30 deg below the horizontal) on both sides and the supine roll maneuver [30] (by $90 \mathrm{deg}$ ) on both sides. Eye movements were recorded and monitored for canalspecific positional nystagmus. Participants were asked to immediately report any sensation of dizziness or vertigo. If the diagnosis of BPPV was established by the pathognomonic nystagmus associated with rotational vertigo, the appropriate liberation maneuvers were performed. The liberation maneuver was performed maximally 2 times on a single day. As many patients suffer from dizziness immediately after repositioning maneuvers, effectiveness was evaluated clinically based on the absence of nystagmus in the subsequent provocation maneuvers (performed immediately after the liberation maneuvers).

Participants in whom BPPV was excluded, underwent a short neuro-otological bedside examination (including ocular motor stability, head impulse test, Romberg testing on solid ground and on foam, bimalleolar vibration sense). Furthermore, a short patient's history was taken, and a review of the available medical documentation was used to identify other causes of their dizziness.

\section{Follow-up visit}

The participants diagnosed with BPPV were approached a third time after 1 to 2 weeks. They were interviewed on the treatments effect as evaluated by the subjective effect on their quality of life. At this follow-up visit, we did not have the opportunity to examine the patients clinically or on the turntable.

\section{Data analysis}

Data analysis was performed using IBM SPSS Statistics 26 (IBM, Armonk, NY, USA). Descriptive statistics are reported as counts/percentages or mean \pm standard deviation as appropriate. Categorical variables are compared using the Fisher's exact test. The yearly estimated prevalence $P_{y}$ of a disease was calculated by:

$$
P_{y}=\frac{365}{D} x P_{d}
$$

where $D$ is the estimated duration of a disease (in days) over the period of 1 year and $P_{d}$ point prevalence of the same disease.

\section{Data availability statement}

Anonymized data used in this study are available upon reasonable request from Dr. S.Y. Bögli.

\section{Results}

Present or recent dizziness was reported by 75 (16.6\%) individuals among the potential study population of 453 residents at the 10 retirement homes in or around the city of Zurich. 68\% were female (mean age: $87.7 \pm 5.6$ years) and $32 \%$ male (mean age: $85.5 \pm 7.8$ ). Of the 71 participants who underwent diagnostic rotations on the manual 2 -axis turntable, $8(=11.3 \%)$ showed the positional nystagmus and rotational vertigo typical of BPPV in one of the provocation maneuvers. The prevalence as well as the estimated yearly prevalence are shown in Fig. 3. In all cases, the Dix-Hallpike maneuver was positive on the left or right side indicating canalolithiasis of the respective posterior canal. If all residents $(N=453)$ correctly indicated whether they suffered from dizziness presently or in the recent past, the point prevalence of BPPV would amount to $1.8 \%$. Based on the questionnaire and the subsequent short neuro-otological bedside examination, most of the remaining $61(=86 \%)$ participants could be assigned to defined diagnoses among which orthostatic dizziness was the most frequent (Table 1).

We asked whether symptoms of dizziness in the participants with BPPV would differ from those with other diagnoses. Time-related properties (e.g., chronic vs. episodic, frequency, duration), triggering factors (e.g., turning in bed) and qualitative attributes (e.g., spinning vertigo, unsteadiness) were all not significantly different between the two groups of participants (Table 2).

Participants, in whom BPPV was diagnosed on the manual 2-axis turntable, were immediately treated with the Epley liberation maneuver [31], whereby the longitudinal body axis was 30 deg below the earth-horizontal and the rotations were performed in 45-deg-steps. In 7 patients a single Epley maneuver to liberate the affected 

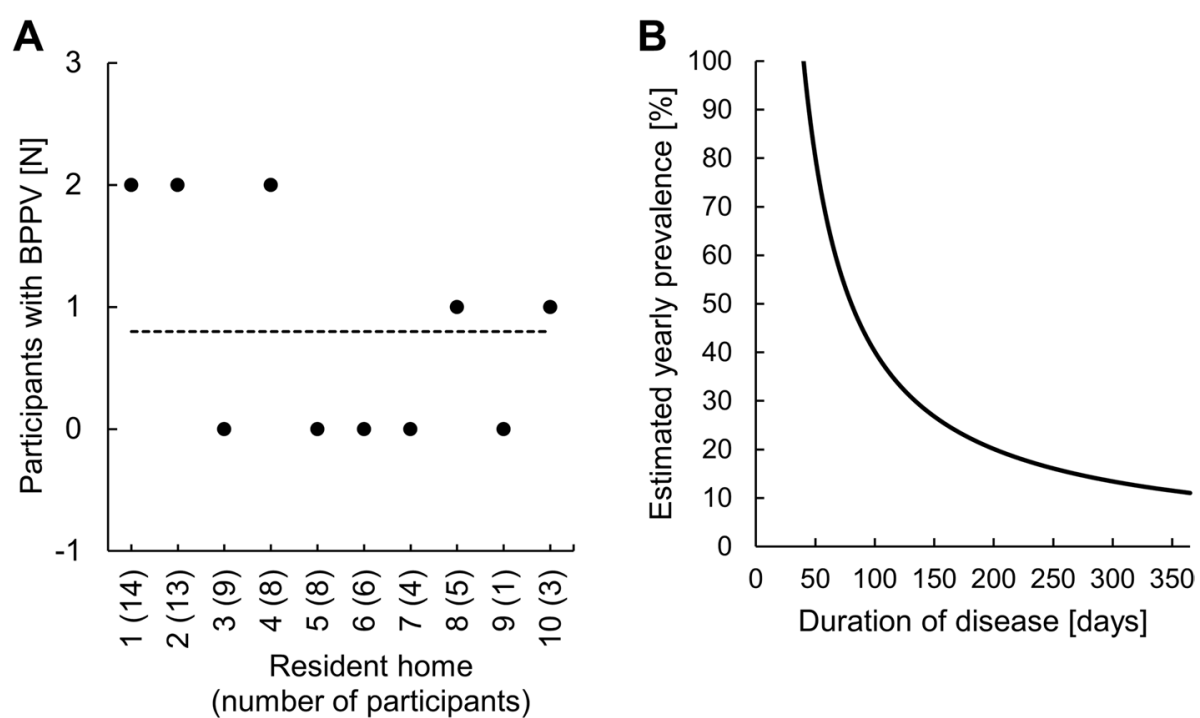

Fig. 3 Point prevalence and estimated yearly prevalence of BPPV. Panel A shows the number of residents per resident home that were diagnosed with BPPV. The dotted line corresponds to the average. Based on the found BPPV point prevalence of $11.3 \%$, panel B depicts estimated BPPV yearly prevalence as a function of BPPV duration (in days) over the period of 1 year (formula given in Methods)

Table 1 Clinical diagnoses

\begin{tabular}{lll}
\hline Primary diagnosis & $\begin{array}{l}\text { Number of } \\
\text { patients }\end{array}$ & $\begin{array}{l}\text { Percentage } \\
\text { of patients }\end{array}$ \\
\hline Orthostatic dizziness & 19 & $26,8 \%$ \\
Multifactorial dizziness & 17 & $23,9 \%$ \\
BPPV & 8 & $11,3 \%$ \\
Proprioceptive dizziness & 3 & $4,2 \%$ \\
Vestibular migraine & 3 & $4,2 \%$ \\
Cardiogenic dizziness & 2 & $2,8 \%$ \\
Unilateral vestibulopathy & 2 & $2,8 \%$ \\
Central vertigo & 2 & $2,8 \%$ \\
Status post BPPV & 2 & $2,8 \%$ \\
Bilateral vestibulopathy & 1 & $1,4 \%$ \\
Psychogenic vertigo & 1 & $1,4 \%$ \\
Meniere's disease & 1 & $1,4 \%$ \\
Unclear cause & 10 & $14,1 \%$ \\
\hline
\end{tabular}

semicircular canal was sufficient with no positional nystagmus and vertigo in the subsequent Dix-Hallpike maneuver. The Epley maneuver had to be repeated in 1 patient to reach absence of positional nystagmus und vertigo.

Participants with diagnosed and treated BPPV were asked about their dizziness 1 to 2 weeks after the "Schwindelbus" visit. 2 individuals were free of dizziness. The other 6 indicated that they were still suffering from dizziness. Of these participants, one reported a decrease of dizzy spells in both frequency and severity, and one an improvement in quality of life.

\section{Discussion}

We visited 10 retirement homes to determine the point prevalence of BPPV among the residents who suffered from dizziness presently or in the recent past. In older patients, diagnostic bedside maneuvers for BPPV (DixHallpike and supine roll maneuvers) are often compromised due to stiffness, pain, and angst, especially among frail patients. For this reason we used a manual 2-axis turntable that was brought by a van ("Schwindelbus") to each resident home where we tested the dizzy residents. By using such a turntable, movements of the head relative to the trunk can be minimized, which considerably reduces the strain on spine, shoulder and trunk during BPPV maneuvers [26].

Among the retirement home residents suffering from dizziness, BPPV was present in $11.3 \%$ at the time of turntable testing. Figure 3 demonstrates the theoretical relation between yearly prevalence and the number of symptomatic days. BPPV is commonly a transient, but frequently recurrent disease. It can only be diagnosed when it is symptomatic. Assuming that BPPV is symptomatic every day of the year, the yearly prevalence would coincide with the point prevalence, in this case $11.3 \%$. On the other hand, assuming (for example) that BPPV among dizzy patients is only symptomatic during 60 days over a year, the yearly prevalence would have to be much higher (i.e., $69 \%$ ) to allow for a point prevalence of $11.3 \%$. There 
Table 2 Univariate analysis of symptoms associated with BPPV

\begin{tabular}{|c|c|c|c|c|}
\hline & & \multicolumn{2}{|c|}{ No. of positive responses } & \multirow{2}{*}{$\begin{array}{l}\text { Fisher } \\
\text { exact } \\
\text { test } \\
\text { (p) }\end{array}$} \\
\hline & & $\begin{array}{l}\text { Without BPPV } \\
(n=63)\end{array}$ & $\begin{array}{l}\text { With BPPV } \\
(n=8)\end{array}$ & \\
\hline \multirow[t]{3}{*}{ Constant dizziness or dizzy spells } & Dizzy spells & 54 & 8 & \multirow[t]{3}{*}{0.59} \\
\hline & Constant dizziness & 8 & 0 & \\
\hline & Not able to answer & 1 & 0 & \\
\hline \multirow[t]{3}{*}{ Duration of dizzy spells } & Seconds to minutes & 38 & 6 & \multirow[t]{3}{*}{0.32} \\
\hline & Hours to days & 14 & 0 & \\
\hline & Not able to answer & 3 & 2 & \\
\hline \multirow[t]{3}{*}{ Frequency of dizzy spells } & Daily & 30 & 5 & \multirow[t]{3}{*}{1.00} \\
\hline & Less often & 22 & 3 & \\
\hline & Not able to answer & 3 & 0 & \\
\hline \multirow[t]{3}{*}{ Between attacks } & Free of dizziness & 44 & 8 & \multirow[t]{3}{*}{0.33} \\
\hline & Less dizzy & 10 & 0 & \\
\hline & Not able to answer & 1 & 0 & \\
\hline \multirow[t]{3}{*}{ Precipitating factor: Backward tilt of the head } & Yes & 18 & 5 & \multirow[t]{3}{*}{0.13} \\
\hline & No & 36 & 3 & \\
\hline & Not able to answer & 9 & 0 & \\
\hline \multirow[t]{3}{*}{ Precipitating factor:Turning in bed } & Yes & 10 & 2 & \multirow[t]{3}{*}{0.27} \\
\hline & No & 43 & 3 & \\
\hline & Not able to answer & 10 & 3 & \\
\hline \multirow[t]{3}{*}{ Precipitating factor: Rising from supine or sitting position } & Yes & 48 & 8 & \multirow[t]{3}{*}{0.34} \\
\hline & No & 11 & 0 & \\
\hline & Not able to answer & 4 & 0 & \\
\hline \multirow[t]{6}{*}{ Characterization of dizziness } & Spinning sensation & 26 & 5 & 0.28 \\
\hline & Staggering or swaying vertigo & 17 & 2 & 1.00 \\
\hline & Light headedness & 13 & 0 & 0.34 \\
\hline & Unstableness & 5 & 1 & 0.53 \\
\hline & General sense of weakness & 2 & 0 & 1.00 \\
\hline & Not able to answer & 2 & 0 & \\
\hline \multirow[t]{3}{*}{ Nausea or vomiting } & Yes & 10 & 2 & \multirow[t]{3}{*}{0.62} \\
\hline & No & 51 & 6 & \\
\hline & Not able to answer & 2 & 0 & \\
\hline \multirow[t]{3}{*}{ Deterioration in the quality of life } & Yes & 35 & 6 & \multirow[t]{3}{*}{0.47} \\
\hline & No & 24 & 2 & \\
\hline & Not able to answer & 4 & 0 & \\
\hline
\end{tabular}

is no way of knowing the number of symptomatic days without testing participants several times during a year, which was beyond the scope of this study. In a representative neuro-otological survey of the general adult population, $8.0 \%$ of patients with moderate or severe dizziness had typical symptoms of BPPV [10], which is somewhat lower than the percentage we found by the actual testing of retirement home residents.

The discrepancy between the BPPV point prevalence of $11.3 \%$ in dizzy retirement home residents, as found in our study, and the BPPV prevalence of $27.6-37.6 \%$ in older patients evaluated at dizziness units $[1,9]$ is probably due to selection, as these studies were performed in hospitals compared to our study that was performed within retirement homes. Only older individuals, in whom the level of dizziness is high enough and outweighs the strain of a transport to the hospital, are evaluated at dizziness units.

Over the potential study population of 453 of the 10 retirement homes the point prevalence of BPPV amounted to $1.8 \%$, which is even higher as the yearly prevalence (1.6\%) in the general population [10]. Again, the point prevalence of $\mathrm{BPPV}$ is most likely considerably lower than the yearly prevalence, which indicates a much higher burden of BPPV on the older population. 
Furthermore, there is a selection bias within our study, as some dizzy patients might have either not been able to answer the questionnaire (and were thus excluded) or were not willing to participate in the study lowering the point prevalence found. Therefore, the percentage of residents who reported present or recent dizziness (16.6\%) needs to be taken with great caution. Structured interviews would need to be conducted among all retirement home residents by investigators with high interrater reliability to determine a reliable percentage of dizzy patients in the population.

All participants with BPPV were successfully treated on the turntable as quantified by the absence of nystagmus in the subsequent control maneuvers. Interesting from a clinical perspective was that time-related properties, triggering factors and qualitative attributes of vertigo or dizziness were not significantly different between the dizzy residents with and those without BPPV. This finding supports the approach to perform diagnostic BPPV maneuvers on every older individual with dizziness irrespective of the presence of a "characteristic" symptom constellation. In spite of the absence of nystagmus in the control maneuvers, only $25 \%$ of residents treated on the turntable were free of dizziness at the follow-up visit. In concordance with the lack of distinct symptoms differentiating residents with and without BPPV, it can be expected that the other residents treated for BPPV were also suffering from other types of dizziness. However, as the study was concluded after the follow-up visit (that did not include a further evaluation of their dizziness origin), this can only be hypothesized.

We conclude that diagnostic BPPV maneuvers are positive in about one tenth of retirement home residents with dizziness or recent dizziness. As BPPV is not always symptomatic, the yearly prevalence is probably higher. In this population, there is a lack of symptoms that clearly differentiate between those with and those without BPPV. This emphasizes the need for a low-threshold implementation of provocation maneuvers. Retirement home residents suffering from dizziness should regularly be tested for BPPV even in the absence of characteristic symptoms. Diagnostic and therapeutic BPPV maneuvers should ideally be performed on a turntable to reduce strain, improve diagnosis and optimize therapy.

\section{Abbreviations}

BPPV: Benign paroxysmal positional vertigo.

\section{Acknowledgements}

Not applicable.

\section{Authors' contributions}

RM acquired the data (including patient evaluation and treatment), analyzed/ interpreted the data, and drafted the manuscript. PZ acquired the data (including patient evaluation and treatment). DS conceived and designed the study, acquired the data (including patient evaluation and treatment), and revised the manuscript for intellectual content. SYB designed the study, acquired the data (including patient evaluation and treatment), analyzed, and interpreted the data, drafted the article, and revised the manuscript for intellectual content. All authors read and approved the final manuscript.

Funding

This project has been supported by a donation from Dr. Hans-Peter Wild to the USZ Foundation.

\section{Availability of data and materials}

The datasets used and/or analyzed during the current study are available from the corresponding author on reasonable request.

\section{Declarations}

\section{Ethics approval and consent to participate}

The study protocol was approved by the local ethics committee (cantonal ethics commission Zurich, BASEC-Nr. 2018-02017) and was in accordance with the ethical standards laid down in the 2013 Declaration of Helsinki for research involving human subjects. Informed consent was obtained from each subject - for both study participation and for publication of identifying images in an online open-access publication.

\section{Consent for publication}

Informed consent was obtained from each subject — for both study participation and for publication of identifying images in an online open-access publication.

\section{Competing interests}

Dominik Straumann is an unpaid consultant of prolim $\mathrm{GmbH}$. The other Authors have no conflicts.

\section{Author details}

${ }^{1}$ Department of Neurology, University Hospital Zurich, Zurich, Switzerland. ${ }^{2}$ University of Zurich, Zurich, Switzerland. ${ }^{3}$ Clinical Neuroscience Center, University Hospital Zurich, Zurich, Switzerland.

Received: 18 October 2021 Accepted: 4 February 2022

Published online: 12 February 2022

\section{References}

1. van Leeuwen RB, Bruintjes TD. Dizziness in the elderly: diagnosing its causes in a multidisciplinary dizziness unit. Ear Nose Throat J. 2014;93(45):162 4, 6-7.

2. Colledge NR, Wilson JA, Macintyre CC, MacLennan WJ. The prevalence and characteristics of dizziness in an elderly community. Age Ageing. 1994;23(2):117-20.

3. Jönsson R, Sixt E, Landahl S, Rosenhall U. Prevalence of dizziness and vertigo in an urban elderly population. J Vestib Res. 2004;14(1):47-52.

4. Ferreira LMBM, Ribeiro KMOF, de Lima KC. Prevalence of dizziness and associated factors in institutionalized elderly persons. J Surg Clin Res. 2015;6(2):45-56.

5. Post RE, Dickerson LM. Dizziness: a diagnostic approach. Am Fam Physician. 2010:82(4):361-89.

6. Straumann D. Bedside examination. Handb Clin Neurol. 2016;137:91-101.

7. Straumann D, Brandt T. Bedside provocation and liberation maneuvers in patients with benign paroxysmal positional Vertigo. Clin Transl Neurosci. 2020;4(1):1.

8. Kim JS, Zee DS. Clinical practice. Benign paroxysmal positional vertigo. N Engl J Med. 2014;370(12):1138-47

9. Geser R, Straumann D. Referral and final diagnoses of patients assessed in an academic vertigo center. Front Neurol. 2012;3:169.

10. von Brevern M, Radtke A, Lezius F, Feldmann M, Ziese T, Lempert T, et al. Epidemiology of benign paroxysmal positional vertigo: a population based study. J Neurol Neurosurg Psychiatry. 2007;78(7):710-5.

11. Baloh RW, Honrubia V, Jacobson K. Benign positional vertigo: clinical and oculographic features in 240 cases. Neurology. 1987;37(3):371-8. 
12. Marciano E, Marcelli V. Postural restrictions in labyrintholithiasis. Eur Arch Otorhinolaryngol. 2002;259(5):262-5.

13. Grimby A, Rosenhall U. Health-related quality of life and dizziness in old age. Gerontology. 1995;41(5):286-98.

14. Tinetti ME, Williams CS, Gill TM. Health, functional, and psychological outcomes among older persons with chronic dizziness. J Am Geriatr Soc. 2000:48(4):417-21.

15. Sixt E, Landahl S. Postural disturbances in a 75-year-old population: I. prevalence and functional consequences. Age Ageing. 1987:16(6):393-8.

16. Ganança FF, Gazzola JM, Ganança CF, Caovilla HH, Ganança MM, Cruz OL. Elderly falls associated with benign paroxysmal positional vertigo. Braz J Otorhinolaryngol. 2010;76(1):113-20.

17. Jumani K, Powell J. Benign paroxysmal positional Vertigo: management and its impact on falls. Ann Otol Rhinol Laryngol. 2017;126(8):602-5.

18. Oghalai JS, Manolidis S, Barth JL, Stewart MG, Jenkins HA. Unrecognized benign paroxysmal positional vertigo in elderly patients. Otolaryngol Head Neck Surg. 2000;122(5):630-4.

19. Maarsingh OR, Dros J, Schellevis FG, van Weert HC, Bindels PJ, Horst HE. Dizziness reported by elderly patients in family practice: prevalence, incidence, and clinical characteristics. BMC Fam Pract. 2010;11:2.

20. Chan K-C, Tsai Y-T, Yang Y-H, Chen P-C, Chang P-H. Osteoporosis is associated with increased risk for benign paroxysmal positional vertigo: a nationwide population-based study. Arch Osteoporos. 2017;12(1):1-9.

21. Yang CJ, Kim Y, Lee HS, Park HJ. Bone mineral density and serum 25-hydroxyvitamin D in patients with idiopathic benign paroxysmal positional vertigo. J Vestib Res. 2017;27(5-6):287-94.

22. Parham K, Kuchel GA. A geriatric perspective on benign paroxysmal positional vertigo. J Am Geriatr Soc. 2016;64(2):378-85.

23. Cakir BÖ, Ercan I, Cakir Z, Civelek Ş, Turgut S. Relationship between the affected ear in benign paroxysmal positional vertigo and habitual headlying side during bedrest. J Laryngol Otol. 2006;120(7):534-6.

24. Prokopakis E, Vlastos I, Tsagournisakis M, Christodoulou P, Kawauchi H, Velegrakis $\mathrm{G}$. Canalith repositioning procedures among 965 patients with benign paroxysmal positional vertigo. Audiol Neurotol. 2013;18(2):83-8.

25. Balatsouras D, Koukoutsis G, Fassolis A, Moukos A, Apris A. Benign paroxysmal positional vertigo in the elderly: current insights. Clin Interv Aging. 2018:13:2251.

26. Bockisch CJ, Straumann D, Weber KP. Curing a 96-year-old patient afflicted with benign paroxysmal positional vertigo on a motorized turntable. Clin Interv Aging. 2014;9:589-91.

27. Liu X, Treister R, Yan Y, Wang H, Li X. Automated mechanical repositioning treatment for posterior canal benign paroxysmal positional vertigo: a single-center experience and literature review. Eur Neurol. 2017;78(5-6):240-6.

28. Shan $X$, Peng $X$, Wang E. Efficacy of computer-controlled repositioning procedure for benign paroxysmal positional vertigo. Laryngoscope. 2015;125(3):715-9.

29. DIX MR, HALLPIKE CS. The pathology symptomatology and diagnosis of certain common disorders of the vestibular system. Proc R Soc Med. 1952;45(6):341-54.

30. McClure JA. Horizontal canal BPV. J Otolaryngol. 1985:14(1):30-5.

31. Epley JM. The canalith repositioning procedure: for treatment of benign paroxysmal positional vertigo. Otolaryngol Head Neck Surg. 1992;107(3):399-404.

\section{Publisher's Note}

Springer Nature remains neutral with regard to jurisdictional claims in published maps and institutional affiliations.

Ready to submit your research? Choose BMC and benefit from:

- fast, convenient online submission

- thorough peer review by experienced researchers in your field

- rapid publication on acceptance

- support for research data, including large and complex data types

- gold Open Access which fosters wider collaboration and increased citations

- maximum visibility for your research: over 100M website views per year

At $\mathrm{BMC}$, research is always in progress.

Learn more biomedcentral.com/submissions 